\title{
Dermatoceratoconjuntivite atópica em pacientes do Ambulatório de Dermatologia Infanto-Juvenil em centro de referência
}

\author{
Atopic keratoconjunctivitis in patients of the pediatric dermatology \\ ambulatory in a reference center
}

\author{
Bernardo Kaplan Moscovici ${ }^{1}$ \\ Andréa Santucci Cesar ${ }^{2}$ \\ Maria Cristina Nishiwaki-Dantas ${ }^{3}$ \\ Silvia A. Soutto Mayor ${ }^{4}$ \\ Alessandra Cristine Marta ${ }^{5}$ \\ Junia Cabral Marques ${ }^{6}$
}

Trabalho realizado no Departamento de Oftalmologia da Irmandade da Santa Casa de Misericórdia de São Paulo - São Paulo (SP) - Brasil.

${ }^{1}$ Estagiário do Setor de Doenças Externas e Córnea do Departamento de Oftalmologia da Irmandade da Santa Casa de São Paulo - São Paulo (SP) - Brasil.

${ }^{2}$ Médica Voluntária Responsável pelo Setor de Alergia Ocular do Departamento de Oftalmologia da Irmandade da Santa Casa de São Paulo - São Paulo (SP) - Brasil.

Doutora, Diretora do Departamento de Oftalmologia da Santa Casa de São Paulo - São Paulo (SP) - Brasil.

${ }^{4}$ Mestre em Medicina, Médica responsável pelo Setor de Dermatologia Pediátrica da Irmandade da Santa Casa de São Paulo - São Paulo (SP) - Brasil.

${ }^{5}$ Médica Dermatologista do Departamento de Dermatologia da Irmandade da Santa Casa de São Paulo - São Paulo (SP) - Brasil.

${ }^{6}$ Médica Assistente Voluntária do Setor de Alergia Ocular do Departamento de Oftalmologia da Irmandade da Santa Casa de São Paulo - São Paulo (SP) - Brasil.

Endereço para correpondência: Bernardo Kaplan Moscovici. Rua das Mangabeiras, 150 - Apto. 81 - São Paulo (SP) CEP 01233-010

E-mail: bernardokaplan@yahoo.com.br

Recebido para publicação em 25.05.2009

Última versão recebida em 10.09.2009

Aprovação em 07.10.2009

Nota Editorial: Depois de concluída a análise do artigo sob sigilo editorial e com a anuência da Dra. Myrna Serapião dos Santos sobre a divulgação de seu nome como revisora, agradecemos sua participação neste processo.

\begin{tabular}{l} 
RESUMO \\
\hline Objetivo: Avaliar a frequência de dermatoceratoconjuntivite atópica, \\
seus sintomas e alterações em pacientes do Ambulatório de Dermatologia \\
Infanto-Juvenil da Irmandade da Santa Casa de Misericórdia de São Paulo \\
com diagnóstico prévio de dermatite atópica. Métodos: Foram avaliados \\
52 portadores de dermatite atópica do Ambulatório de Dermatologia \\
Infanto-Juvenil, menores de 16 anos (média de idade $8,9 \pm 4$ entre 2 e \\
16 anos) por meio de um protocolo de questões de sintomas e avaliação \\
oftalmológica dos sinais. Os sinais e sintomas foram graduados de \\
0 (ausente) a 4 (maior intensidade), e em alguns casos apenas como \\
presentes ou não. A análise foi descritiva e estatística, com nível de \\
significância de 5\%. Resultados: A frequência da dermatoceratocon- \\
juntivite atópica foi de 76,9\% entre os 52 pacientes com dermatite \\
atópica, dos quais $26,7 \%$ com conjuntivite atópica e $50,2 \%$ com blefarite \\
atópica. No grupo de dermatite atópica encontramos maior número de \\
pacientes do sexo feminino e no grupo conjuntivite atópica maior número \\
de pacientes do sexo masculino. A idade foi praticamente igual nos \\
grupos estudados. Os sintomas mais frequentes foram olho vermelho e \\
prurido, principalmente no grupo de conjuntivite atópica. Este último foi \\
o sintoma com escore mais intenso e presentes em todos os pacientes \\
do grupo conjuntivite atópica. Papilas e blefarite foram os sinais mais \\
encontrados. O tempo de ruptura do filme lacrimal encontrava-se alterado \\
na maioria dos pacientes. Conclusões: A frequência de dermatocerato- \\
conjuntivite atópica foi de $76,9 \%$ nos pacientes com dermatite atópica. \\
O prurido foi o sintoma mais frequente, seguido por olhos vermelhos, \\
mais relacionados ao grupo de conjuntivite atópica. A blefarite e as \\
papilas foramos sinais mais encontrados, também no grupo deconjuntivite \\
atópica. Devido ao crescimento da prevalência da dermatite atópica na \\
população infantil, seria prudente realizar a avaliação oftalmológica de \\
rotina desses pacientes, visto que nenhum dos pacientes de nosso estudo \\
estavam em acompanhamento oftalmológico prévio. \\
\hline
\end{tabular}

Descritores: Conjuntivite alérgica; Dermatite atópica; Blefarite; Ceratoconjuntivite; Doenças da córnea

\section{INTRODUÇÃ̃O}

Dermatite atópica (DA) é uma doença inflamatória crônica da pele, que ocorre mais comumente entre a infância e a adolescência e resulta em 
defeitos na barreira de proteção da pele, do sistema imunológico e respostas exacerbadas a antígenos microbianos e alérgenos ${ }^{(1-8)}$.

A prevalência da DA é de 10 a $20 \%$ em crianças nos EUA, Europa, Japão e Austrália, semelhante à do Brasil, onde é de $13,2 \%$. Nos adultos, a prevalência diminui para 1 a $3 \%$, mais frequente em mulheres ${ }^{(4-5)}$.

Não existe teste laboratorial específico ou característica clínica patognomônica para o seu diagnóstico, que é baseado em critérios clínicos, dos quais o mais utilizado é o de Hanifin e Rajka ${ }^{(4,9)}$.

A dermatoceratoconjuntivite atópica (DCCA) foi primeiramente descrita em 1952 por Hogan, e é definida como lesão inflamatória crônica, não infecciosa, da superfície ocular, que os portadores de DA podem sofrer em qualquer etapa do curso da doença dermatológica e que independe do seu grau de gravidade segundo alguns autores, ou como doença de conjuntiva e das pálpebras, segundo outros ${ }^{(10-17)}$.

A DCCA afeta entre 15 e $67,5 \%$ dos pacientes com DA. É mais frequente no sexo masculino, com início geralmente entre a segunda e a quinta década de vida, com pico de incidência a partir de 40 anos, embora tenham sido descritos casos em crianças $^{(9-10,12,17-21)}$.

O diagnóstico da DCCA é clínico, seguindo alguns parâmetros como prurido, diagnóstico prévio de DA e lesões típicas oculares ${ }^{(22)}$.

Com a progressiva urbanização da população, nas últimas décadas ocorreram alterações ambientais que elevaram a prevalência de atopia, e consequentemente da DA na população. O impacto econômico nos EUA causado pela alergia ocular é grande; com gastos de mais de 200 milhões de dólares com colírios antialérgicos na última década ${ }^{(22)}$.

O objetivo do nosso estudo foi avaliar a frequência de DCCA, seus sintomas e alterações do segmento anterior em pacientes do Ambulatório de Dermatologia Infanto-Juvenil da Irmandade da Santa Casa de Misericórdia de São Paulo (ISCMSP), com diagnóstico prévio de dermatite atópica.

\section{MÉTODOS}

Estudo prospectivo, observacional e transversal, realizado no Ambulatório de Alergia Ocular da ISCMSP, aprovado pelo Comitê de Ética em Pesquisa sob protocolo nº 291/08

\section{Grupo de estudo}

Foram avaliados, no período de março de 2006 a julho de 2008, no Ambulatório de Alergia Ocular, os pacientes do Ambulatório de Dermatologia Infanto-Juvenil com diagnóstico clínico de dermatite atópica, segundo os critérios de Hanifin e Rajka, que obedeceram aos seguintes critérios de inclusão e exclusão:

Critérios de inclusão: diagnóstico clínico de dermatite atópica, idade menor que 16 anos, acompanhados por representante legal, assinatura do termo de ciência e consentimento livre por representante legal.
Critérios de exclusão: usuários de lente de contato, cirurgia ocular prévia.

Foram selecionados, de acordo com os critérios mencionados, 52 portadores de DA, $23(44,2 \%)$ do sexo masculino e 29 $(55,8 \%)$ do sexo feminino.

Os pacientes apresentaram idades entre 2 e 16 anos, com média de 8,9 e desvio padrão de 4,1 anos.

$\mathrm{O}$ autor aplicou um protocolo de questões com os seguintes itens: prurido, olho vermelho, lacrimejamento, sensação de corpo estranho, secreção e fotofobia, que foram graduados de 0 (ausência) a 4 (maior intensidade).

Todos os pacientes foram avaliados de acordo com a seguinte sequência por examinador que não tinha conhecimento prévio das respostas dos pacientes ao questionário anterior:

- Exame externo: foram avaliados eczema palpebral e perda de cílios, que foram classificados em presentes ou ausentes.

- Biomicroscopia: foram avaliados os seguintes sinais: blefarite, hiperemia conjuntival, papilas conjuntivais, papilas gigantes (maiores que $1 \mathrm{~mm}$ ), folículos conjuntivais, atrofia conjuntival, pontos de Horner-Trantas, ceratite, ulcerações corneais e opacidade lenticular.

- Tempo de ruptura do filme lacrimal (TRFL)

Os sinais biomicroscópicos foram graduados de 0 (ausência) a 4 (maior intensidade), com exceção da atrofia conjuntival, pontos de Horner-Trantas e alterações do cristalino, que foram classificados em presentes ou ausentes.

\section{Análise estatística}

Utilizamos o teste qui-quadrado de Pearson, o teste exato de Fisher e o teste $t$ de Student, considerando como estatisticamente significante $\mathrm{p}<0,05$.

\section{RESULTADOS}

Após avaliação clínica, esses pacientes foram classificados em dois grupos: com e sem DCCA (pacientes que não se encaixaram em outros grupos citados). O grupo com DCCA foi subdividido em dois grupos:

- Grupo 1: pacientes com DCCA que necessitaram de tratamento com colírio antialérgico, caracterizado por conjuntivite atópica.

- Grupo 2: pacientes com DCCA que necessitaram apenas de tratamento com lubrificante ocular e higiene palpebral com xampu neutro, apresentando apenas blefarite atópica.

Quarenta pacientes (76,9\%) tinham DCCA no nosso estudo, dos quais $15(26,7 \%)$ pertenciam ao grupo 1 e $25(50,2 \%)$ ao grupo 2 .

Encontramos no grupo de DCCA 55\% de mulheres e $45 \%$ de homens, já o grupo 1 era composto por $33,3 \%$ de mulheres e $66,7 \%$ de homens. Finalmente no grupo 2 encontramos $68 \%$ de mulheres e $32 \%$ de homens, sendo essa diferença estatisticamente significante pelo teste Qui-quadrado de Pearson ( $\mathrm{p}=0,033)$. 
O gráfico 1 mostra a distribuição dos escores dos sintomas referidos pelos pacientes segundo a mesma divisão anterior.

$\mathrm{O}$ prurido foi referido em todos os pacientes do grupo $1 \mathrm{e}$ em $72 \%$ dos pacientes do grupo 2 . Olho vermelho foi referido em $86,7 \%$ dos pacientes do grupo 1 e em $44,4 \%$ dos pacientes do grupo 2. Os outros sintomas foram menos referidos e em menor intensidade.

Os gráficos 2 e 3 exibem a distribuição dos escores dos sinais blefarite e papilas encontrados pelo examinador segundo a mesma divisão anterior.

Em relação aos demais sinais encontrados no grupo conjuntivite atópica, 6 pacientes apresentaram ceratite, 2 papilas gigantes, 1 atrofia conjuntival, 4 eczema palpebral, 3 perda dos cílios e 1 pontos de Horner-Trantas (Figura 1).

No grupo blefarite atópica, 12 pacientes apresentavam papilas e 1 ceratite. Nesse grupo, poucos achados foram identificados como intensos.

Nenhum dos pacientes avaliados apresentou ulceração de córnea ou opacidade lenticular.

O TRFL foi alterado em $54,5 \%$ dos pacientes do grupo $2 \mathrm{e}$ em $33,3 \%$ dos pacientes do grupo 1 , porém essa diferença foi estatisticamente insignificante $(\mathrm{p}=0,297)$,

Encontramos diferença estatisticamente significante quando relacionamos prurido e papilas, $\operatorname{com}(\mathrm{p}=0,008)$ pelo teste Exato de Fisher.

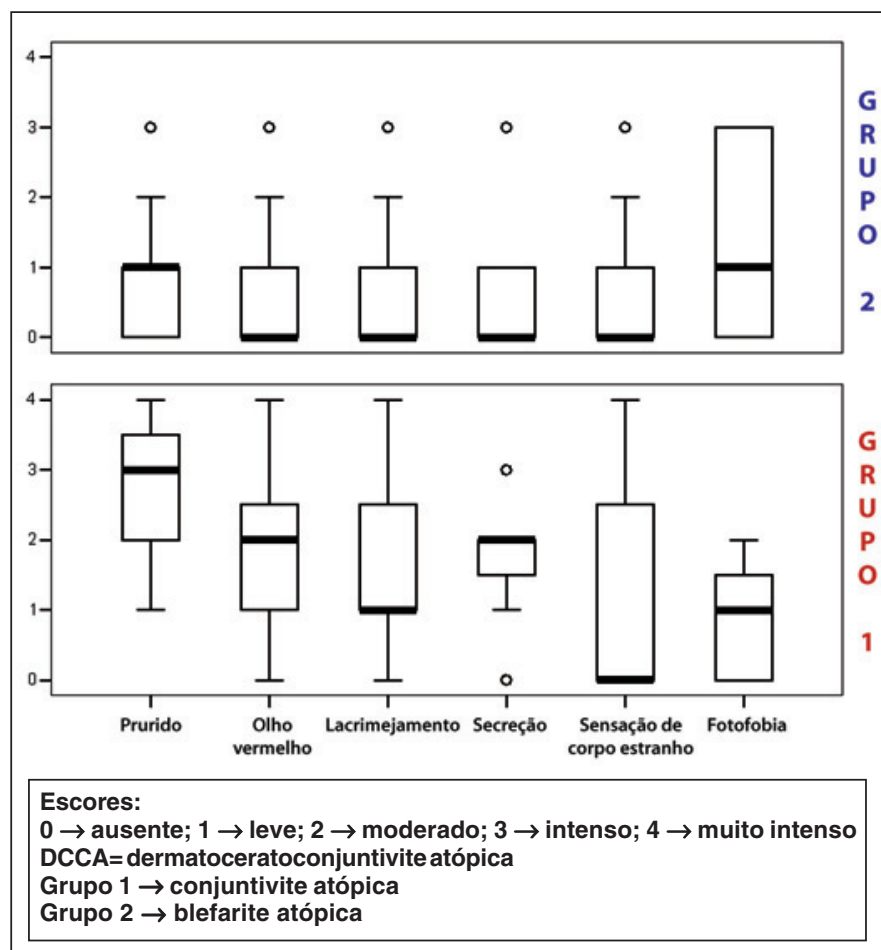

Fonte: Departamento de Oftalmologia, Irmandade da Santa Casa de Misericórdia de São Paulo, 2008

Gráfico 1 - Box Plot com a distribuição dos sintomas nos grupos diagnósticos portadores de dermatite atópica do Ambulatório de Dermatologia Infanto-Juvenil da Irmandade da Santa Casa de Misericórdia de São Paulo
Realizamos o teste de Fisher para avaliar se a distribuição dos sinais e sintomas entre os grupos diagnósticos foi diferente. Para isso agrupamos os escores:

Até 1 - Escore 0 a 1

2 ou mais - Escore 2 a 4

As papilas foram mais evidentes no grupo 1 , com escores mais elevados $(\mathrm{p}<0,001)$.

A secreção foi mais evidente no grupo 1, com escore 2 ou mais $(\mathrm{p}<0,001)$. O mesmo ocorreu com hiperemia e prurido $(\mathrm{p}=0,006$ e $\mathrm{p}<0,001$, respectivamente).

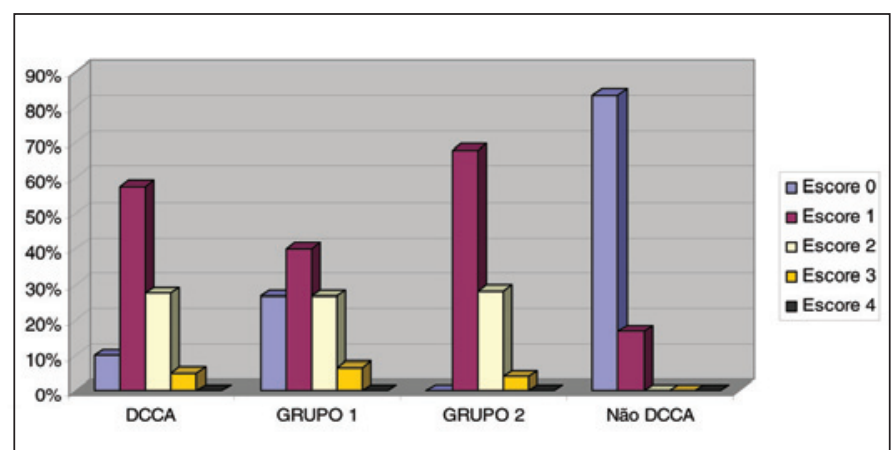

Escores:

$0 \rightarrow$ ausente; $1 \rightarrow$ leve; $2 \rightarrow$ moderado; $3 \rightarrow$ intenso; $4 \rightarrow$ muito intenso DCCA $=$ dermatoceratoconjuntivite atópica

Grupo $1 \rightarrow$ conjuntivite atópica

Grupo $2 \rightarrow$ blefarite atópica

Fonte: Departamento de Oftalmologia, Irmandade da Santa Casa de Misericórdia de São Paulo, 2008

Gráfico 2 - Distribuição da blefarite nos grupos diagnósticos portadores de dermatite atópica do Ambulatório de Dermatologia Infanto-Juvenil da Irmandade da Santa Casa de Misericórdia de São Paulo

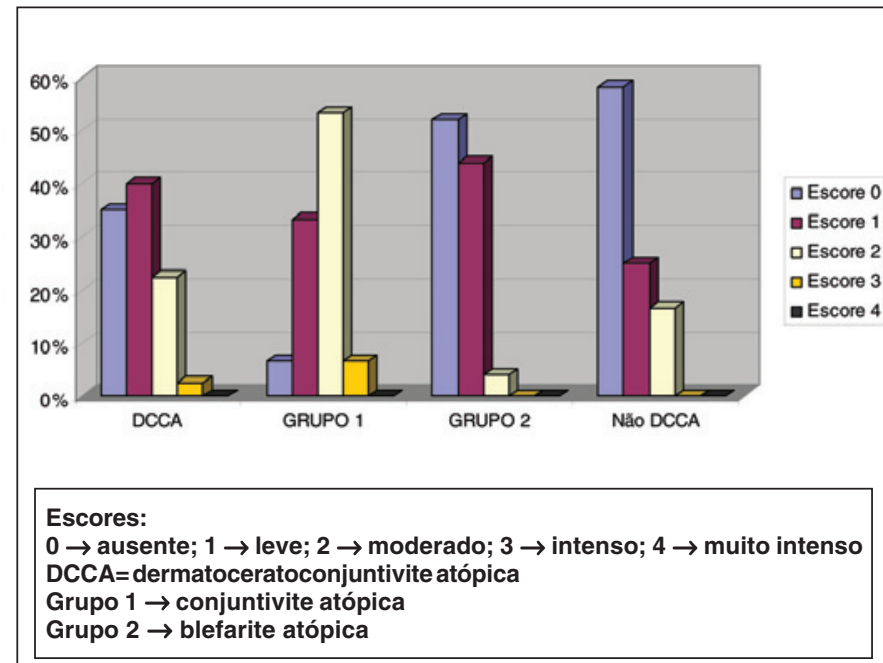

Fonte: Departamento de Oftalmologia, Irmandade da Santa Casa de Misericórdia de São Paulo, 2008

Gráfico 3 - Distribuição das papilas nos grupos diagnósticos portadores de dermatite atópica do Ambulatório de Dermatologia Infanto-Juvenil da Irmandade da Santa Casa de Misericórdia de São Paulo 


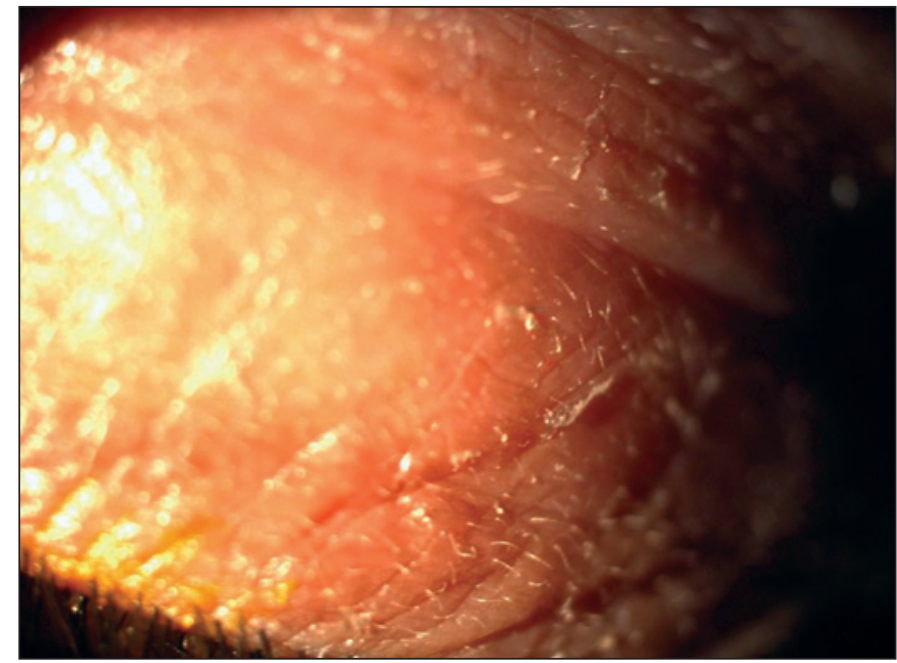

Figura 1 - Eczema palpebral

\section{DISCUSSÃO}

Um dos poucos estudos que analisam a frequência de DCCA em crianças com DA foi realizado por Carmi et al. ${ }^{(23)}$, na França, que encontraram $25,4 \%$ de crianças portadoras de doença oftalmológica em um estudo com 59 pacientes e idade média 36,2 meses. No nosso estudo, apenas 3 pacientes do grupo DCCA tinham menos de 3 anos, dificultando a comparação dos nossos dados. Se compararmos com os pacientes do grupo 1, encontramos dados similares (26,7\%); o mesmo acontece se confrontarmos nossos dados com a descrição de Ortega ${ }^{(24)}$ de $21 \%$ de DCCA em crianças.

Uchio et al. ${ }^{(25)}$, em um estudo, no Japão, com 216 pacientes com idade média de 24,8 $\pm 7,8$ anos, encontraram frequência de $32,4 \%$ de doença oftálmica do segmento anterior.

Dogru et al. ${ }^{(14)}$, em outro estudo no Japão, com 362 pacientes com idade média de 22,7 anos, encontraram frequência semelhante à do nosso estudo, de $67,5 \%$, apesar da diferença de idade.

A DCCA apresenta espectro variável de frequência e crescimento nos últimos anos, podendo levar a números subesti$\operatorname{mados}^{(12,15,21-22)}$. Os estudos no Japão de Uchio ${ }^{(25)}$ e Dogru ${ }^{(14)}$, quando comparados, mostram números destoantes. Essa diferença pode estar relacionada à priorização de apenas alterações mais graves da DCCA por Uchio e cols (conjuntivite atópica), que encontrou taxas menores da doença.

Alguns autores, como Uchio et al..$^{(9)}$, Marback et al. ${ }^{(26)} \mathrm{e}$ Calonge et al. ${ }^{(12)}$, estudaram graduações clínicas para a DCCA e outras alergias, assim padronizando os estudos e facilitando as discussões. Dessa maneira, poderíamos correlacionar com mais coerência os dados encontrados em diversos estudos.

Com relação ao sexo, encontramos maior número de pacientes do sexo feminino $(55,8 \%)$ com DA; entretanto, encontramos maior quantidade do masculino no grupo 1 sendo essa diferença estatisticamente significante e concordante com o padrão encontrado na literatura( ${ }^{(4,15-17,24-25)}$.
Não houve diferença estatisticamente significante entre as idades nos pacientes com DCCA, esses dados concordam com os da literatura, em que os casos mais graves estão relacionados à maior duração do processo alérgico ${ }^{(15-17,23,25,27)}$.

Existem poucos estudos quantificando os sintomas por meio de questionários na literatura, o que torna escassa a comparação dos dados. Um deles foi realizado por Marback et al. ${ }^{(26)}$ com idade média 14,6 anos $( \pm 7,0)$, que confrontou os achados oculares e sintomas dentre as diversas conjuntivites alérgicas e encontraram maior frequência de prurido, que foi significantemente relacionado à DCCA. Nesse mesmo estudo, foi verificada a relação dos sintomas com a DCCA e a CCP, sendo o lacrimejamento mais relacionado a esta última. No nosso estudo, esse item foi pouco referido. No estudo de Marback et al., eles também correlacionaram a intensidade do prurido, sensação de corpo estranho, lacrimejamento e fotofobia com a ceratite e verificaram correlação com todos. Em nosso estudo, a fotofobia foi pouco referida, mais intensamente no grupo blefarite atópica, diferença essa, entretanto, estatisticamente insignificante. Em contrapartida, olho vermelho foi maior no grupo conjuntivite atópica $(\mathrm{p}=0,006)$.

Analisando os sinais oftalmológicos (Gráficos 2 e 3), o mais encontrado foi a blefarite, presente em $73 \%$ dos pacientes, seguida pelas papilas, vistas em $58,6 \%$ dos pacientes. Foster et al. ${ }^{(15)}$ relataram taxa de $55,6 \%$ de blefarite, já Tuft et al. ${ }^{(17)} 33 \%$. Dogru et al. ${ }^{(14)}$ referiram $67,5 \%$ de papilas e Power et al. ${ }^{(27)} 65 \%$, em seguimento de 3 anos de 20 pacientes com idade média de 45,6 anos. Carmi et al. ${ }^{(23)}$ encontraram $18 \%$ de papilas na sua amostra, provavelmente devido à faixa etária de 36,2 meses.

A grande maioria dos pacientes do nosso estudo apresentou achados oftálmicos mais amenos da DCCA, mesmo os que foram classificados no grupo 1 . As papilas estavam presentes em todos os casos do mesmo grupo, mas também em boa parte do grupo 2. Sua presença não denotava gravidade do quadro, mas talvez sua intensidade pudesse ser um fator de risco para doença mais intensa, visto que ela foi mais encontrada no grupo conjuntivite atópica $(\mathrm{p}<0,001)$.

Foster et al. ${ }^{(15)}$, Tuft et al. ${ }^{(17)}$, Dogru et al. ${ }^{(14)}$, Power et al. ${ }^{(27)}$, Uchio et al. ${ }^{(25)}$ encontraram em seus estudos maior quantidade de papilas gigantes, atrofia conjuntival, perda de cílios e ceratite que em nosso estudo. Esses sinais foram pouco encontrados e apenas no grupo conjuntivite atópica (grupo 1). A diferença entre esses estudos e o nosso é explicada pela amostra dos estudos acima ser composta por pacientes mais graves, com um processo alérgico crônico.

Dogru et al. ${ }^{(14)}$ encontraram $62 \%$ de alteração no TRFL, e alguns outros autores ${ }^{(14,24)}$ concluíram que a alteração lacrimal nesses pacientes pode ser devida à deficiência na produção de mucina e pelo dano celular resultante da reação alérgica e formação de papilas.

Asano-Kato et al. ${ }^{(28-29)}$ estudaram a evaporação da lágrima em portadores de DA e acharam evaporação maior, principalmente correlacionada com a intensidade de blefarite. No nos- 
so estudo, não encontramos associação entre alteração do TRFL e blefarite.

A literatura convenciona como mais frequente a DA em crianças, porém a DCCA é mais encontrada a partir da quarta década de vida, quando a DA começa a diminuir sua frequên$\operatorname{cia}^{(4,9,15-16,21)}$. No nosso estudo, verificamos alta taxa de DCCA, principalmente casos leves (blefarite atópica). No entanto, encontramos número elevado de pacientes com conjuntivite atópica, se considerarmos que o acometimento se torna mais frequente a partir da vida adulta e a gravidade da doença está relacionada com a cronicidade do processo alérgico.

Como citado anteriormente, a prevalência de DA e DCCA vem aumentando progressivamente. Na última década, o gasto financeiro com essa doença foi elevado ${ }^{(6)}$, de modo que é de vital importância a avaliação frequente desses pacientes por oftalmologistas, mesmo com idades mais baixas, visando melhorar a qualidade de vida destes pacientes e obter menor índice de complicações oculares, visto que nenhum dos pacientes presentes no estudo estava em acompanhamento oftalmológico.

\section{CONCLUSÕES}

A frequência de DCCA foi de $76,9 \%$, dos quais $26,7 \%$ com conjuntivite atópica e 50,2\% com blefarite atópica.

Foi encontrado maior número de pacientes do sexo feminino com DA e maior número do masculino com conjuntivite atópica. Não houve diferença de idade entre os grupos.

O sintoma mais referido foi o prurido, seguido por olho vermelho. Os sinais mais encontrados foram blefarite e papilas.

\section{ABSTRACT}

Purpose: To evaluate the frequency of atopic keratoconjunctivitis, its symptoms and changes in patients of Pediatric Dermatology Service of "Santa Casa de Misericordia de São Paulo" Hospital with a previous diagnosis of atopic dermatitis. Methods: Fifty-two patients with atopic dermatitis under 16 years old (mean age $8.9 \pm 4.1$ between 2 and 16 years old) were evaluated through a protocol of questions and assessment of signs and symptoms. The signs and symptoms were graded from 0 (absent) to 4 (highest intensity), and in some cases only as present or not. The analysis was descriptive and statistical, with a 5\% significance level. Results: The frequency of atopic keratoconjunctivitis was $76.9 \%$ among the 52 patients with atopic dermatitis, including atopic conjunctivitis (26.7\%) and atopic blepharitis (50.2\%). Atopic dermatitis is more frequent in female patients and atopic conjunctivitis in male. Age was practically the same in both groups. The most frequent symptoms were red eye and itching, especially in the atopic conjunctivitis group. Itching was the most intense symptom and was present in all patients of the atopic conjunctivitis group. Blepharitis and papillae were the most com- mon signs. Other signs were less frequent. The tear break-up time was changed in most patients. Conclusions: The prevalence of atopic keratoconjunctivitis was $76.9 \%$ in patients with atopic dermatitis. Itching was the most frequent symptom, followed by red eye, in patients with atopic conjunctivitis. Blepharitis and papillae were the most frequent signs in patients with atopic conjunctivitis. Due to the increasing prevalence of atopic dermatitis in children, it would be prudent to perform a routine ophthalmologic evaluation of these patients. From the patients evaluated in this study, none had been previously monitored with ophthalmological exams.

Keywords: Conjunctivitis, allergic; Dermatitis, atopic; Blepharitis; Keratoconjunctivitis; Corneal diseases

\section{REFERÊNCIAS}

1. Abelson MB, Granet D. Ocular allergy in pediatric practice. Curr Allergy Asthma Rep. 2006;6(4):306-11.

2. Sehgal VN, Jain S. Atopic dermatitis: ocular changes. Int J Dermatol. 1994; 33(1):11-5. Review.

3. Eiseman AS. The ocular manifestations of atopic dermatitis and rosacea. Curr Allergy Asthma Rep. 2006;6(4):292-8.

4. Wolff K, Goldsmith LA, Katz SI, Gilchrest BA, Paller AS, Leffell DJ, editors. Fitzpatrick's dermatology in general medicine. $7^{\text {th }}$ ed. New York: McGraw-Hill; 2008. p.147-58.

5. Harper J, Oranje A, Prose NS, editors. Textbook of pediatric dermatology. $2^{\text {nd }}$ ed. Massachusetts: Wiley-Blackwell; 2006. p.179-296

6. Nakata K, Inoue Y, Harada J, Maeda N, Watanabe H, Tano Y, Shimomura Y, et al. A high incidence of Staphylococcus aureus colonization in the external eyes of patients with atopic dermatitis. Ophthalmology. 2000;107(12):2167-71.

7. Nivenius E, van der Ploeg I, Jung K, Chryssanthou E, van Hage M, Montan PG. Tacrolimus ointment vs steroid ointment for eyelid dermatitis in patients with atopic keratoconjunctivitis. Eye. 2007;21(7):968-75.

8. Peuravuori H, Kari O, Pletonen S, Aho VV, Saari JM, Collan Y et al. Group IIA phospolipase A2 content of tears in patients with atopic blepharoconjunctivitis. Graefes Arch Clin Exp Ophthalmol. 2004;242(12):986-9.

9. Uchio E, Kimura R, Migita H, Kozawa M, Kadonosono K. Demographic aspects of allergic ocular diseases and evaluation of new criteria for clinical assessment of ocular allergy. Graefes Arch Clin Exp Ophthalmol. 2008;246(2):291-6.

10. Barney NP. Vernal and atopic keratoconjunctivitis. In: Krachmer JH, Mannis MJ, Holland EJ, editors. Cornea: fundamentals, diagnosis and management. $2^{\text {nd }}$ ed. St. Louis: Mosby; 2005. v.1. p.670-3.

11. Bonini S. Atopic keratoconjunctivitis. Allergy. 2004;59(Suppl 78):71-3.

12. Calonge M, Herreras JM. Clinical grading of atopic keratoconjunctivitis. Curr Opin Allergy Clin Immunol. 2007;7(5):442-5.

13. Calonge M. Ocular allergies: association with immune dermatitis. Acta Ophthalmol Scand Suppl. 2000;(230):69-75.

14. Dogru M, Nakagawa N, Tetsumoto K, Katakami C, Yamamoto M. Ocular surface disease in atopic dermatitis. Jpn J Ophtalmol. 1999;43(1):53-7.

15. Foster CS, Calonge M. Atopic keratoconjunctivitis. Ophthalmology. 1990; 97(8):992-1000.

16. Hogan MJ. Atopic keratoconjunctivitis. Trans Am Ophthalmol Soc. 1952;50: 265-81.

17. Tuft SJ, Kemeny DM, Dart JK, Buckley RJ. Clinical features of atopic keratoconjunctivitis. Ophthalmology. 1991;98(2):150-8.

18. Belfort R, Marbeck P, Hsu CC, Freitas D. Epidemiological study of 134 subjects with allergic conjunctivitis. Acta Ophthalmol Scand Suppl. 2000;(230):38-40.

19. Goulart DA, Goulart DG, Cypel MC, Dantas PEC, Nishiwak-Dantas MC. Perfil clínico-epidemiológico de pacientes do Ambulatório de Alergia Ocular da Santa Casa de São Paulo. Arq Bras Oftalmol. 2003;66(5):609-15.

20. Nishiwaki-Dantas MC. Manual de alergia ocular. São Paulo: Phoenix; 2006. p. 58

21. Nishiwaki-Dantas MC, Felberg DMS. Conjuntivite atópica. In: Höfling-Lima AL, Nishiwaki-Dantas MC, Alves MR, organizadores. Doenças externas oculares e córnea. Rio de Janeiro: Cultura Médica; 2008. p.173-82. 
22. Bielory L, Katelaris CH, Lightman S, Naclerio RM. Treating the ocular component of allergic rhinoconjunctivitis and related eye disorders. Med Gen Med. 2007;9(3):35.

23. Carmi E, Defossez-Tribout C, Ganry O, Cene S, Tramier B, Milazzo S, Lok C. Ocular complications of atopic dermatitis in children. Acta Derm Venereol. 2006;86(6):515-7.

24. Zepeda Ortega B, Rosas Vargas MA, Ito Tsuchiya FM, Del Rio Navarro BE, Sienra Monge JJ. Conjuntivitis alérgica en la infancia. Rev Alerg Mex. 2007; 54(2):41-53.

25. Uchio E, Miyakawa K, Ikezawa Z, Ohno S. Systemic and local immunological features of atopic dermatitis patients with ocular complications. Br J Ophthalmol. 1998;82(1):82-7.
26. Marback PMF, Freitas D, Paranhos Junior A, Belfort Junior R. Aspectos clínicos e epidemiológicos da conjuntivite alérgica em serviço de referência. Arq Bras Oftalmol. 2007;70(2):312-6.

27. Power WJ, Tugal-Tutkun I, Foster CS. Long-term follow-up of patients with atopic keratoconjunctivitis. Ophthalmology. 1998;105(4):637-42.

28. Asano-Kato N, Fukagawa K, Tsubota K, Urayama K, Takahashi S, Fujishima $\mathrm{H}$. Quantitative evaluation of atopic blepharitis by scoring of eyelid conditions and measuring the water content of the skin and evaporation from the eyelid surface. Cornea. 2001;20(3):255-9.

29. Asano-Kato N, Fukagawa K, Takano Y, Kawakita T, Tsubota K, Fujishima H, Takahashi S. Treatment of atopic blepharitis by controlling eyelid skin water retention ability with ceramide gel application. Br J Ophthalmol. 2003;87(3):362-3. 